\title{
The value of dynamic contrast-enhanced MRI in the diagnosis and management of triple-negative breast cancer
}

\author{
Heba Azzam ${ }^{1 *}$ D, Rasha Kamal ${ }^{1}$, Hany El-Assaly ${ }^{1}$ and Liza Omer ${ }^{2}$
}

\begin{abstract}
Background: Breast cancer is undoubtedly the world's most frequent cancer among women. Triple-negative breast cancer (TNBC) is a subtype of breast cancer that does not express estrogen, progesterone, or human epidermal growth factor receptors, yet its imaging is considered a challenge to radiologists having imprecise features. In this study, we aimed at defining the MRI characteristics of triple-negative breast cancer to validate its impact on management.

Results: Most of the TNBCS in this study showed malignant pattern kinetic curves (types II and III), 87/104 (83.7\%), and $P$ value 0.673 (not significant), and regarding the type of lesion enhancement, 104/172 cases (60.5\%) showed mass enhancement compared to 52/172 (30.2\%) non-mass enhancement and 16/172 (9.3\%) focus enhancement, $P$ value 0.185 (not significant). As for the internal enhancement pattern of mass lesions, rim internal enhancement was predominant in TNBC mass lesions, 48/104 (46.2\%), as well as intratumoral bright signal intensity on T2-weighted images, 108/172 (62.8\%), P value 0.001 (highly significant).
\end{abstract}

Conclusion: Triple-negative breast cancers (TNBC) are larger, better defined, and more necrotic than conventional cancers. On MRl, necrosis yields high T2-weighted signal intensity.

Keywords: Dynamic contrast-enhanced MRI, Triple-negative breast cancer

\section{Background}

Breast cancer $(\mathrm{BC})$ is without a doubt the world's most frequent cancer among women, and it is the commonest reason of cancer related death in women globaly [1].

Triple-negative breast cancer (TNBC) is a subtype of breast cancer which does not express estrogen receptors (ER), progesterone receptors (PR), and human epidermal growth factor receptor 2 (HER2). They represent nearly $12-26 \%$ of total breast cancers, and are coupled with an extra aggressive clinical course and poor prognosis. They have an elevated rate of local recurrence and metastatic disease, with a 5 -year survival of more or less $75 \%[2,3]$. The frequency of TNBC is high in younger, habitually premenopausal women $[4,5]$.

Imaging of TNBC is considered a diagnostic challenge to breast radiologists. On mammography and ultrasound,

\footnotetext{
* Correspondence: heba.m.azzam@gmail.com

'Diagnostic and Interventional Radiology, Cairo University, Maadi, Cairo, Egypt

Full list of author information is available at the end of the article
}

triple-negative cancer shows imprecise characteristics that can imitate lesions with benign features [6]. Patients with TNBC are commonly youthful with BRCA gene mutation, and they have dense breasts which additionally lowers mammographic sensitivity and specificity. The speedy progression of the TNBC which is not coupled with architectural distortions makes early detection of the disease exceptionally hard [7].

Breast MRI screening is beneficial for patients with high-risk and those with occult breast carcinoma, which has a rather higher prevalence of TNBC $[8,9]$. MRI shows malignant characteristics that might not be recognized using mammography or ultrasound; consequently, it is considered of higher precision in detecting the local extent of disease in recently diagnosed breast cancer $[6$, 10]. TNBC presented quite a lot of MRI predictors on dynamic contrast-enhanced magnetic resonance imaging (DCE-MRI); these could be valuable in identifying TNBC from other breast cancer subtypes [5]. 
Neo-adjuvant chemotherapy (NAC) has been the customary treatment for locally advanced breast cancer. It is mostly designed to decrease the tumor size, thus allowing breast-conserving surgery. Other privileges of NAC are that systemic therapy may be started earlier, and it permits evaluation of a patient's response to chemotherapy [11].

Therefore, the aim of this work is to assess the DCEMRI features of triple-negative breast cancer and to validate its impact on management.

\section{Methods}

The study is a retrospective study in which we analyzed the contrast-enhanced MRI morphology descriptors and kinematic curve in 100 patients with triple-negative breast cancer.

\section{Data collection}

Demographic and clinical data including patient's name, age, clinical presentation, family history, and histopathological diagnosis as well as imaging data including breast ultrasound and mammography were compiled.

\section{MRI protocol}

Dynamic MR imaging was performed with high field strength (1.5 T MRI system) [Panorama; Philips Medical Systems, Netherland] using a bilateral breast surface coil with the patient in the prone position. Total study time ranged from 30 to $45 \mathrm{~min}$. No sedation was used. - Comfort of the patients was ensured, and they were asked to remain still during the whole examination.

\section{Imaging acquisition protocol}

A- Cases were examined first by pre-contrast sequences:

Axial T1-weighted turbo spin echo (TR/TE $=500 /$

$5.3 \mathrm{msec}$ ), sagittal and axial T2-weighted images using turbo spin echo $(\mathrm{TR} / \mathrm{TE}=120 / 4.9 \mathrm{msec})$, axial short time inversion recovery (STIR) $(\mathrm{TR} / \mathrm{TE}=80 /$ $6.5 \mathrm{msec}$ ), and a pre-contrast fat-saturated T2weighted pulse sequence.

B- Contrast-enhanced sequences/series:

A bolus of contrast was injected manually intravenous, and enhanced images were obtained immediately after contrast agent injection.

C- Post processing:

Subtraction was used to highlight the enhancing features in the image.

1. Quantitative analysis was done by using time/ signal intensity curve. A color overlay map was displayed, indicating regions of threshold enhancement.
2. The maximum intensity projection (MIP) was performed to demonstrate the distribution of disease in the breast in relation to the skin, nipple, chest wall, and large vessels.

\section{Image analysis}

Magnetic resonance images were reviewed by two radiologists in the same setting and the final diagnosis was reached by their agreement (in consensus). The findings of dynamic contrast-enhanced MRI study were evaluated in accordance with the ACR BI-RADS MRI Lexicon, 2013, that includes two major categories of descriptors: morphology and enhancement kinetics.

\section{Morphology descriptors}

Enhancing lesions were categorized as focus, mass, and non-mass enhancement.

1- Focus/foci A focus is a breast lesion smaller than 5 $\mathrm{mm}$.

2- Mass A mass is a 3-dimensional space-occupying lesion.

a. Multiplicity of masses (Fig. 1).

b. Mass was characterized by:

Shape (oval, round, irregular), margin

(circumscribed, not circumscribed), and internal enhancement characteristics (homogenous, heterogenous, rim enhancement, dark internal septations).

3- Non-mass enhancement (Fig. 2)

a. Distribution pattern:

- Focal area

- Linear enhancement.

- Segmental enhancement

- Regional enhancement

- Diffuse enhancement

b. Internal enhancement characteristics:

- Homogenous

- Heterogeneous

- Clumped

- Clustered ring

\section{Associated features}

Associated findings with or without enhancement include nipple retraction (when the areola is distorted more than nipple, might be circumferential pulling in but the nipple usually visible unless it is very late stage) or inversion (slit-like and can be averted often results from duct ectasia or fibrosis), skin retraction, thickening or invasion, lymphadenopathy, pectoral muscle invasion and chest wall invasion, architectural distortion, high signal intensity in ducts on unenhanced images, abnormal signal void, hematoma, edema, and cysts were reported. 

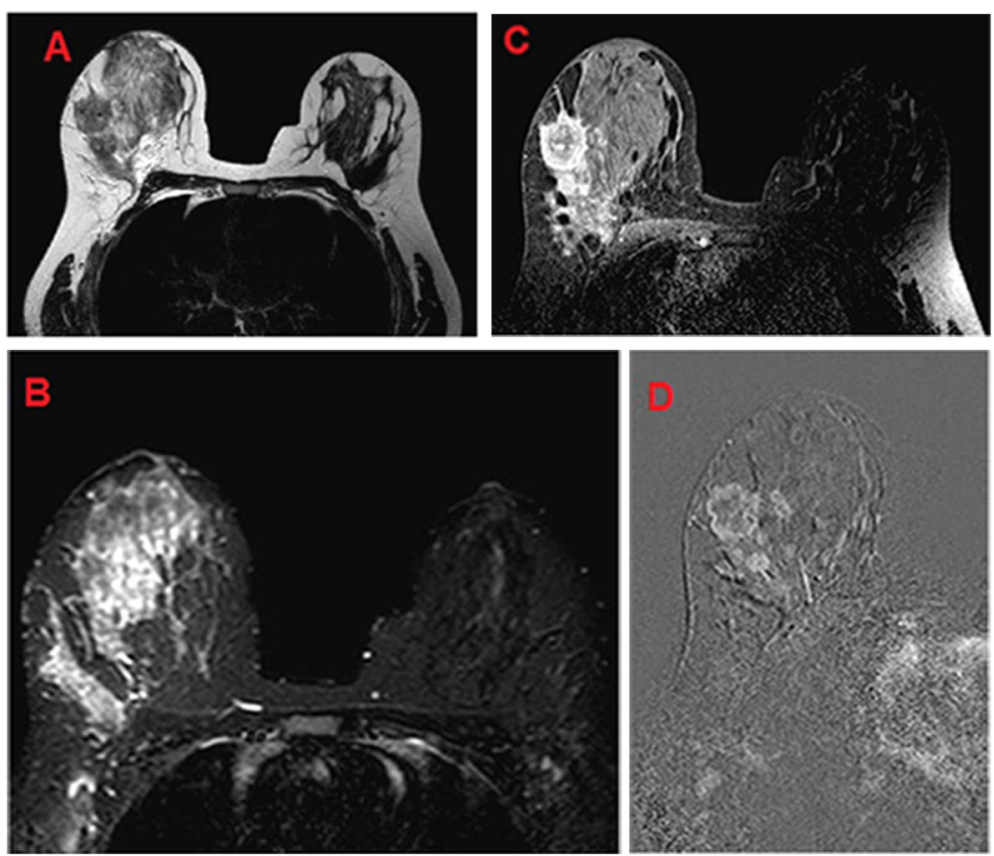

Fig. 1 MR images: a T2. b STIR. c DCE-MRI post-contrast. d DCE-MRI subtraction images. A large right upper outer quadrant (UOQ) irregular mass with a speculated margin is seen eliciting intermediate T2 and high STIR signal intensity "arrow" with multiple foci surrounding the lesion are also seen displaying intermediate T2 and bright STIR signal intensity "arrowheads." On dynamic post-contrast images the enhancing mass "arrow" and the multiple peri-lesional foci all show rim enhancement "arrowheads" The pathology revealed TN invasive ductal carcinoma

\section{Signs of spread of malignancy}

On the MR images of the breast, spreading to the axillary region, other lymph nodes, the chest wall muscles, the ribs, the adjacent pleura and lung, or overlying skin was reported.

\section{Kinetic enhancement curve assessment}

Three enhancement patterns were identified on the basis of the time/signal intensity curve: types I, II, and III.

All patients were assigned a BI-RADS category [6] according to the ACR MRI BI-RADS lexicon.

\section{Statistical analysis}

The data was summarized using number and percentage for qualitative variables, mean, and SD for normally distributed quantitative variables.

Comparison between groups was done using chisquare test for qualitative variables and independent $t$ test for quantitative variables.

$P$ value less than or equal to 0.05 was considered statistical significant, and 0.01 or less considered highly significant.

\section{Results}

The study included 100 patients with 172 pathologically proved TNBC.

\section{Age}

Ages of TNBC patients ranged from 24 to 60 years (mean age $44.04 \pm \mathrm{SD}$ ).

\section{Clinical presentation}

Most of patients presented with palpable mass lesions (80/100, 80\%).

\section{Breast density}

- $12 / 100$ (12\%) had almost entirely fatty breast density; 56/100 (56\%) had scattered fibroglandular tissue; 32/100 (32\%) had heterogenous fibroglandular tissue; no cases $(0 / 100,0 \%)$ had extreme fibroglandular tissue.

\section{Background parenchymal enhancement (BPE)}

- 40/100 (40\%) minimal BPE; 36/100 (36\%) mild BPE; 24/100 (24\%) moderate BPE.

\section{Type of lesion enhancement}

TNBC (172 lesions) distributed as:

104/172 (60.5\%) showed mass enhancement. 52/172

(30.2\%) showed non-mass enhancement. 16/172 (9.3\%) showed focus enhancement. 

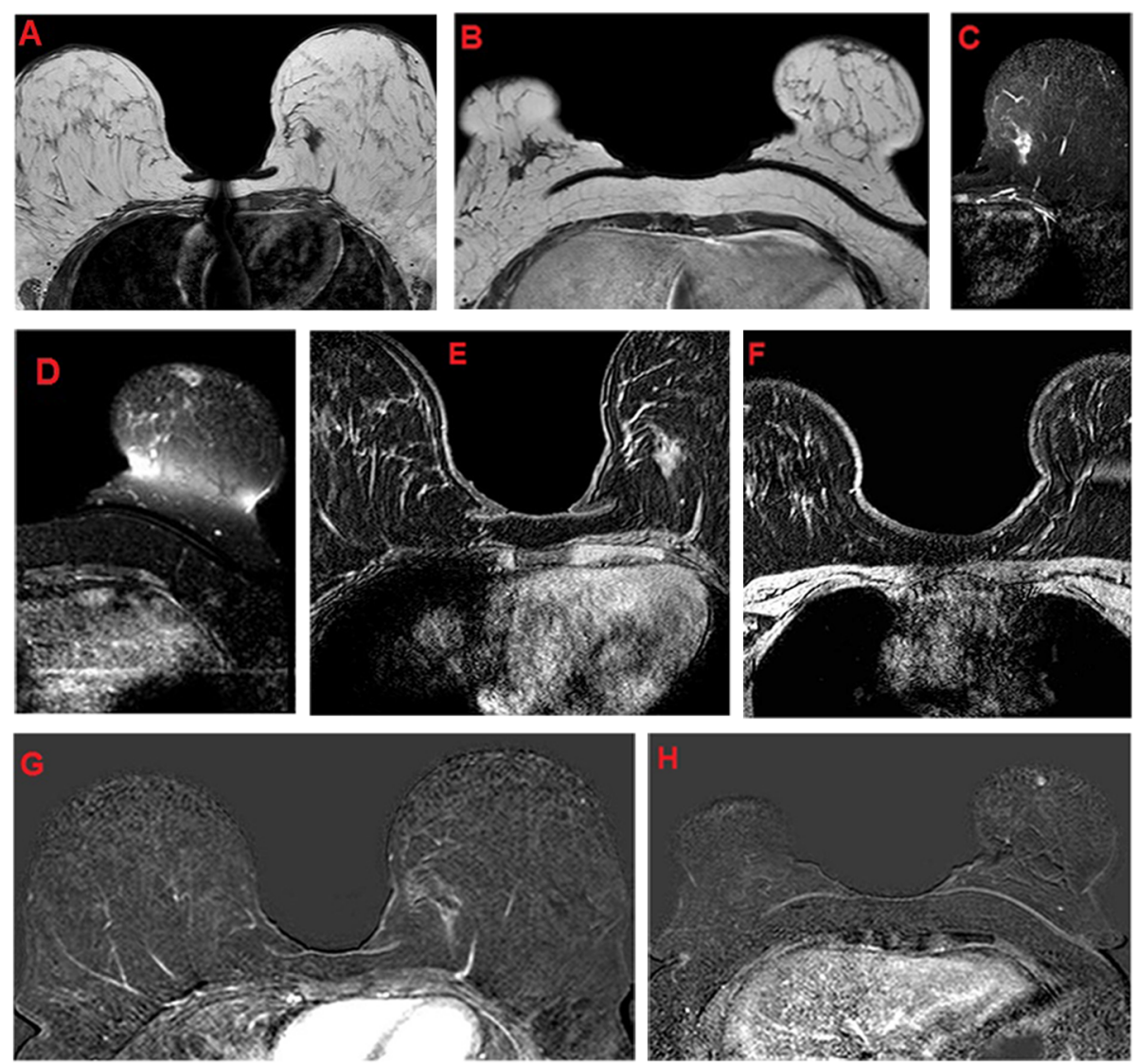

Fig. 2 MR images: a, b T2. c, d STIR. e, f DCE-MRI post-contrast. $\mathbf{g}$, h DCE-MRI subtraction images. Left LIQ lesion "arrow" eliciting intermediate T2 and high STIR signal intensity and showing focal non-mass enhancement on dynamic post-contrast images. Another left lower central small rounded mass with a circumscribed margin "arrowhead" eliciting intermediate T2 and STIR signal intensity shows homogenous enhancement in post-contrast images The pathology revealed TN invasive ductal carcinoma

Internal enhancement characteristics:

The predominant internal enhancement pattern of the TNBCs is rim enhancement. 24/104 (23.1\%) mass lesions showed homogenous enhancement. 32/ 104 (30.8\%) mass lesions showed heterogeneous enhancement. 48/104 (46.2\%) mass lesions showed rim enhancement.

\section{Time/signal intensity curve (K-curve)}

- 47/104 (45.2\%) lesions showed washout (type III) curve; 40/104 (38.5\%) lesions showed plateau (type II) curve; 17/104 (16.3\%) lesions showed persistent (type I) curve.

- 25/52 (48.1\%) lesions showed washout (type III) curve; 21/52 (40.4\%) lesions showed plateau (type II) curve; 6/52 (11.5\%) lesions showed persistent (type I) curve.

\section{T 2 signal intensity}

108/172 (62.8\%) lesions elicited high T2 signal intensity; 60/172 (34.9\%) lesions elicited low T2 signal intensity; 4/ $172(2.3 \%)$ lesion was indistinct in T2 weighted images. While 148/172 (86\%) lesions elicited high T2 STIR signal intensity, 20/172 (11.6\%) lesions elicited low T2 STIR signal intensity and 4/172 (2.3\%) lesion was indistinct in T2 STIR weighted images. The increased T2 and STIR signal is due to presence of necrotic areas which elicits high signal on T2 WI.

\section{Shape of the mass lesions}

40/104 (38.5\%) were rounded or oval in shape, and 64/ 104 (61.5\%) were irregular in shape.

\section{Histopathological diagnosis}

After revision of core biopsy reports, we found that:

TNBC: $80 / 100(80 \%)$ cases were invasive ductal carcinoma. 16/100 (16\%) cases were lobular carcinoma. 4/100 (4\%) case was mucoid carcinoma. 


\section{Discussion}

Although TNBC accounts for a fairly little minority of breast cancer cases, it is responsible for a quite large share of breast cancer deaths, due to the commonly aggressive clinical course and the lack of successful targeted therapies $[7,12]$.

Triple-negative phenotype is characterized by distinct clinical, histopathological, and molecular characteristics, and is associated with the development of recurrence, distant metastases, and a poorer prognosis; therefore, better understanding of the imaging features of TN cancers, especially at MRI, provides an imaging biomarker with clinical implications.

This study aimed to determine the dynamic contrastenhanced magnetic resonance imaging features of TN breast cancers in addition to MRI features that are more frequent with triple-negative breast cancer subtypes such as round shape mass lesion, mass with rim enhancement in post contrast images, and intratumoral bright signal intensity on T2-weighted images, while still maintaining other DCE-MRI parameters commonly seen with other breast cancer subtypes as distribution of fibroglandular tissue (FGT) and BPE, mass margin, non-mass enhancement distribution and enhancement pattern, lymph nodes status, and types of kinetic curves.

In this study, the patients' age ranged from 24 to 60 years (mean age $44.04 \pm \mathrm{SD}$ ) which agrees with $\mathrm{Li}$ and Han [9] as well as Schmadeka et al. [13] who stated that TNBC more commonly affects youthful patients $(<50$ years).

Similar to previous reports as Reis-Filho and Tutt [14], Sung et al. [4], Schmadeka et al. [13], and Abramson et al. [15], our study results showed that TNBCs in $80 \%$ cases were invasive ductal carcinoma.

In our study, $12 / 100(12 \%)$ had almost entirely fatty breast density, 56/100 (56\%) had scattered fibroglandular tissue, 32/100 (32\%) had heterogeneous fibroglandular tissue, no cases $(0 / 100,0 \%)$ had extreme fibroglandular tissue which is in opposition to Boisserie-Lacroix et al. [7] who reported that TNBC patients are usually young and have dense breasts as well as Kim et al. [16] who reported that women with high mammographic breast density (MBD) were less likely to have triple-negative breast cancer.

$104 / 172(60.5 \%)$ of our cases presented as mass lesions. This goes in concordance with Dogan et al. [17], $\mathrm{Li}$ and Han [9], and Osman et al. [5] who stated that TNBC most often presents as mass enhancement.

As regards the shape of mass lesions in this study, 40/ $104(38.5 \%)$ were rounded or oval in shape and 64/104 (61.5\%) were irregular in shape; however, Sung et al. [4] found that the majority of TNBC lesions were lobulated and Ivanac et al. [18] as well as Osman et al. [5] found most TNBC were rounded and or oval masses.
We found that most masses had irregular or speculated margins $(72 / 104,69.2 \%)$ while $32 / 104(30.8 \%)$ had smooth (circumscribed) margins compared to those of Uematsu et al. [19] whose findings which showed that $39 \%$ of the TNBCs had smooth margins. On the other hand, Youk et al. [6] and Osman et al. [5] reported that a smooth mass margin tended to be prominently associated with TNBC.

Rim internal enhancement was predominant in TNBC mass lesions $(48 / 104,46.2 \%)$ in this study with $24 / 104$ (23.1\%) homogenous enhancement and 32/104 (30.8\%) heterogeneous enhancement. Our results are consistent with many studies $[5-7,16,19,20]$. Teifke et al. [20] declared that rim enhancement is the most useful MR feature for identifying TNBC and rim enhancement is associated with higher grade tumors.

The majority of TNBC mass lesions demonstrated areas of high T2 intratumoral signal intensity (108/172, 62.8\%). Our result is compatible with Sung et al. [4] and Osman et al. [5] who stated that high T2 signal intensity was significantly associated with TN cancer and that intratumoral high signal intensity on unenhanced fat suppressed T2-weighted images corresponded morphologically and pathologically to intratumoral necrosis.

Most of the patients in this study showed malignant pattern kinetic curves (types II and III), (87/104, 83.3\%) This result is similar to many studies as Chen et al. [21], Dogan et al. [17], and Youk et al. [6] and unlike Uematsu et al. [19] that reported a persistent enhancement pattern was significantly associated with TNBC.

In this study, TNBC were diagnosed by DCE-MRI in all cases, and this coincides with previous studies such as Dogan et al. [17] who studied the characteristics of TNBC by mammography, ultrasound, and MR imaging; TNBC were detected by MRI in all cases and in $91 \%$ and $93 \%$ of cases by mammography and ultrasound, respectively. Also, Schmadeka et al. [13] suggested that MRI is the most sensitive (99-100\%).

\section{Conclusion}

In conclusion, in this study, TNBC presented several characteristic MRI features on DCE-MRI such as multiple lesions, mass enhancing type, irregular shape, not circumscribed margin, rim enhancement, high T2 signal intensity, and malignant kinetic curve pattern.

\section{Abbreviations}

ACR: American College of Radiology; BC: Breast cancer; BI-RADS: Breast Imaging-Reporting and Data System; BPE: Background parenchymal enhancement; BRCA gene: Breast cancer gene; DCE-MRI: Dynamic contrastenhanced magnetic resonance imaging; ER: Estrogen receptor; FGT: Fibroglandular tissue; HER2: Human epidermal growth factor receptor 2; MIP: Maximum intensity projection; NAC: Neo-adjuvant chemotherapy; PR: Progesterone receptor; TNBC: Triple-negative breast cancer; UOQ: Upper outer quadrant 


\section{Acknowledgements}

Not applicable.

\section{Funding}

Not applicable (no funding received for this study).

\section{Availability of data and materials}

All the datasets used and analyzed during this study are available with the corresponding author on reasonable request.

\section{Authors' contributions}

All authors have read and approved the manuscript. HA, RK, HE, and LO contributed equally to this work. HA and RK designed the research. HA and LO performed the research. HE and RK analyzed the data. HA and HE wrote the paper.

\section{Ethics approval and consent to participate}

This study was approved by the research ethics committee of the Radiology department of the Faculty of medicine Cairo University on 3/9/2014, Reference number of approval: 780-2014.

All patients included in this study gave a written informed consent to participate in the research. If the patient was less than 16 years old, or unconscious at the time of study, written informed consent was given by their parent or legal guardian.

\section{Consent for publication}

All patients included in this study gave a written informed consent to publish the data contained in this study. If the patient was less than 16 years old, or unconscious at the time of study, written informed consent was given by their parent or legal guardian.

\section{Competing interests}

The authors declare that they have no competing interests.

\section{Author details}

${ }^{1}$ Diagnostic and Interventional Radiology, Cairo University, Maadi, Cairo, Egypt. ${ }^{2}$ Cairo University, Cairo, Egypt.

Received: 27 September 2019 Accepted: 21 January 2020

Published online: 04 February 2020

\section{References}

1. Ferlay J, Soerjomataram I, Ervik M, Dikshit R, Eser S, Mathers C, Rebelo M, Parkin DM, Forman D, Bray F (2013). IARC CancerBase no. 11) GLOBOCAN 2012 v1.0, cancer incidence and mortality worldwide. International Agency for Research on Cancer, Lyon

2. Krizmanich-Conniff KM, Paramagul C, Patterson SK, Helvie MA, Roubidoux MA, Myles JD, Jiang K, Sabel M (2012) Triple receptor- negative breast cancer: imaging and clinical characteristics. AJR Am J Roentgenol 199(2): 458-464

3. Golden DI, Lipson JA, Telli ML, Ford JM, Rubin DL (2013) Dynamic contrastenhanced MRI-based biomarkers of therapeutic response in triple-negative breast cancer. J Am Med Inform Assoc 20(6):1059-1066

4. Sung JS, Jochelson MS, Brennan S, Joo S, Wen YH, Moskowitz C, Zheng J, Dershaw DD, Morris EA (2013) MR imaging features of triple-ngative breast cancers. Breast J 19(6):643-649

5. Osman NM, Chalabi N, Abd Raboh NM (2014) Triple negative breast cancer: MRI features in comparison to other breast cancer subtypes with correlation to prognostic pathologic factors. Egypt J Radiol Nucl Med 45(4):1309-1316

6. Youk JH, Son EJ, Chung J, Kim JA, Kim EK (2012) Triple-negative invasive breast cancer on dynamic contrast-enhanced and diffusionweighted MR imaging: comparison with other breast cancer subtypes. Eur Radiol 22(8): 1724-1734

7. Boisserie-Lacroix M, MacGrogan G, Debled M, Ferron S, Asad-Syed M, McKelvie-Sebileau P, Mathoulin-Pélissier S, Brouste V, Hurtevent-Labrot G (2013) Triple-negative breast cancers: associations between imaging and pathological findings for triple-negative tumors compared with hormone receptor-positive/human epidermal growth factor receptor-2-negative breast cancers. Oncologist 18(7):802-811

8. Dogan BE, Turnbull LW (2012) Imaging of triple-negative breast cancer. Ann Oncol 23(6):23-29
9. Li J, Han X (2014) Research and progress in magnetic resonance imaging of triple-negative breast cancer. Magn Reson Imaging 32(4):392-396

10. Atkins JJ, Appleton CM, Fisher CS, Gao F, Margenthaler JA (2013) Which imaging modality is superior for prediction of response to neoadjuvant chemotherapy in patients with triple negative breast cancer? J Oncol: 964863, 7 pages. https://doi.org/10.1155/2013/964863

11. Kawashima H, Inokuchi M, Furukawa H, Ikeda H, Kitamura S (2014) Magnetic resonance imaging features of breast cancer according to intrinsic subtypes: correlations with neoadjuvant chemotherapy effects. SpringerPlus 3:240

12. Podo F, Buydens LM, Degani H, Hilhorst R, Klipp E, Gribbestad IS, Van Huffel S, van Laarhoven HW, Luts J, Monleon D, Postma GJ, Schneiderhan-Marra N, Santoro F, Wouters H, Russnes HG, Sørlie T, Tagliabue E, Børresen-Dale AL, FEMME Consortium (2010) Triple-negative breast cancer: present challenges and new perspectives. Mol Oncol 4(3):209-229

13. Schmadeka R, Harmon BE, Singh M (2014) Triple-negative breast carcinoma current and emerging concepts. Am J Clin Pathol 141(4):462-477

14. Reis-Filho JS, Tutt AN (2008) Triple negative tumours: a critical review. Histopathology 52(1):108-118

15. Abramson VG, Lehmann BD, Ballinger TJ, Pietenpol JA (2015) Subtyping of triple-negative breast cancer: implications for therapy. Cancer 121(1):8-16

16. Kim MY, Choi N, Yang JH, Yoo YB, Park KS (2015) Background parenchymal enhancement on breast MRI and mammographic breast density: correlation with tumour characteristics. Clin Radiol. https://doi.org/10.1016/j.crad.2015. 02.017 Article in press

17. Dogan BE, Gonzalez-Angulo AM, Gilcrease M, Dryden MJ, Yang WT (2010) Multimodality imaging of triple receptor-negative tumors with mammography, ultrasound, and MRI. AJR Am J Roentgenol 194(4):1160-1166

18. Ivanac G, Dominkovic MD, Brkljacic B, Zagreb HR, Orasje BA Imaging features of triple negative breast carcinoma: mammography, ultrasound and MRI. Eur Soc Radiol. https://doi.org/10.1594/ecr2014/C-0482 ECR 2014/C-0482

19. Uematsu T, Kasami M, Yuen S (2009) Triple-negative breast cancer: correlation between MR imaging and pathological findings. Radiology 250(3):638-647 (c) RSNA

20. Teifke A, Behr O, Schmidt M, Victor A, Vomweg TW, Thelen M, Lehr HA (2006) Dynamic MR imaging of breast lesions: correlation with microvesse distribution pattern and histologic characteristics of prognosis. Radiology 239(2):351-360

21. Chen JH, Agrawal G, Feig B, Baek HM, Carpenter PM, Mehta RS, Nalcioglu O, Su MY (2007) Triple negative breast cancer: MRI features in 29 patients. Ann Oncol 18(12):2042-2043

\section{Publisher's Note}

Springer Nature remains neutral with regard to jurisdictional claims in published maps and institutional affiliations.

\section{Submit your manuscript to a SpringerOpen ${ }^{\circ}$ journal and benefit from:}

- Convenient online submission

- Rigorous peer review

- Open access: articles freely available online

High visibility within the field

- Retaining the copyright to your article

Submit your next manuscript at $\boldsymbol{\nabla}$ springeropen.com 